\title{
MedienPädagogik
}

Zeitschrift für Theorie und Praxis der Medienbildung

\section{Editorial: Qualitative Forschung in der Medienpädagogik}

\author{
Horst Niesyto und Heinz Moser
}

Seit den 1980er-Jahren haben sich qualitative Forschungsansätze in den Sozialwissenschaften stark verbreitet. In der Medienpädagogik ist dies nicht zuletzt mit einer Hinwendung zu subjektorientierten Ansätzen und Methoden verbunden, die den Fokus der Forschung auf den aktiven Umgang der Menschen mit Medien legen. Seither haben sich die Zugänge und Konzepte der qualitativen Forschung stark differenziert

- etwa mit Entwicklungen wie:

- die Herausbildung konkreter Forschungsprogramme wie grounded theory,

- die Diskussionen um Gütekriterien der qualitativen Forschung,

- die Diskussion zur Verbindung von qualitativen und quantitativen Methoden (Triangulation),

- die Entwicklung von Programmen zur qualitativen Daten- und Inhaltsanalyse,

- der Hinwendung zu ethnographischen Verfahren,

- der verstärkte Einbezug visueller Methoden (z.B. Zeichnungen, Foto, Video),

- die Erneuerung von Konzepten der Aktions- und Praxisforschung,

- eine Betonung dekonstruktiver Ansätze, die insbesondere die Position der Forschenden thematisieren.

In der Medienpädagogik ist mitunter auch argumentiert worden, dass der Stand der methodologischen Entwicklung seit den 1990er Jahren stagniere. Es ist Zeit für die Diskussion der Frage, welche innovativen Verfahren die methodologische Diskussion weiterführen könnten. Das Themenheft «Qualitative Forschung in der Medienpädagogik» geht auf Vorträge auf einer gleichnamigen Tagung zurück, die von der Kommission Medienpädagogik der Deutschen Gesellschaft für Erziehungswissenschaft (DGfE) am 22. und 23. März 2007 an der Pädagogischen Hochschule Ludwigsburg veranstaltet wurde.

Die Beiträge von Barbara Stauber, Peter Holzwarth und Björn Maurer und Stefan Welling basieren auf medienpädagogischen Praxisforschungsprojekten, in denen jeweils Kinder/Jugendliche die Möglichkeiten hatten, im Rahmen medienpädagogischer Settings Medien für Selbstausdruck und/oder Kommunikation zu nutzen.

In dem Beitrag über «Gender-Dynamiken in der Rekonstruktion von Bildungsprozessen in Medienprojekten», der auf die Evaluation des Programms «Jugend und verantwortungsvolle Mediennutzung» (Landesstiftung Baden-Württemberg) zurückgeht, stellt Barbara Stauber den qualitativen Teil der Evaluationsstudie vor. Im Mittelpunkt steht die Frage, inwieweit die jeweilige Methoden-Kombination für die Gender-Perspektive ertragreich war und inwieweit es gelang, Bildungsprozesse in den

Niesyto, Horst, und Heinz Moser. 2008. «Editorial: Qualitative Forschung in der Medienpädagogik». MedienPädagogik 14, (Januar), i-iv. https:// doi.org/10.21240/mpaed/14/2008.00.00.X. 
untersuchten medienpädagogischen Projekten unter der Perspektive von GenderDynamiken zu erfassen. Barbara Stauber arbeitet u.a. die Bedeutung von narrativen Interviews mit Jugendlichen für die Reflexion ihrer Medienpraxis heraus.

Der Beitrag von Peter Holzwarth und Björn Maurer zu dem Thema «Erfahrungen an der Schnittstelle von Medienarbeit und Praxisforschung» reflektiert die Verbindung von praktischer Medienarbeit und Forschung am Beispiel des EU-Forschungsprojekts "Children in Communication About Migration» (CHICAM). Nach der Darstellung des Projektkontextes und des medienpädagogischen Konzepts werden die Aufgaben und Kompetenzen der medienpädagogischen sowie der wissenschaftlichen Begleitung dargestellt. Die Autoren verdeutlichen, dass es gerade bei Praxisforschungsprojekten wichtig ist, die verschiedenen Rollen klar zu definieren, damit die Forschung hinreichend Distanz zu den beobachteten Prozessen einnehmen und auch die Interaktionen zwischen medienpädagogischer und wissenschaftlicher Begleitung reflektieren kann.

Auch der Beitrag von Stefan Welling bezieht sich auf ein medienpädagogisches Praxisforschungsprojekt, das in der Offenen Jugendarbeit angesiedelt war. Im Mittelpunkt seiner Fallstudien steht die Frage nach den Potenzialen einer milieusensitiven computerunterstützten Jugendarbeit. Stefan Welling rekonstruiert hierfür mittels der Methode der Gruppendiskussion - in Anlehnung an Bohnsack - medienpädagogische Orientierungsmuster bei JugendarbeiterInnen. Anhand von zwei ausgewählten Fallbeispielen werden verschiedene Orientierungsmuster herausgearbeitet, die für Jugendliche unterschiedliche Partizipationsmöglichkeiten im Umgang mit Medien eröffnen. Die Analysen verstehen sich als ein Beitrag zur bislang wenig entwickelten medienpädagogischen Professionsforschung.

Der Beitrag von Ulrike Wagner und Helga Theunert über «Konvergenzbezogene Medienaneignung in Kindheit und Jugend» stellt das Konzept der konvergenzbezogenen Medienaneignung vor und gibt einen Überblick zu den bisherigen Grundlagenstudien, die das JFF (Institut für Medienpädagogik in Forschung und Praxis) im Zeitraum von 2001 bis 2007 durchführte. Der Beitrag konzentriert sich dabei auf die Reflexion von Forschungsperspektiven, die dem vernetzten Medienhandeln von Kindern und Jugendlichen unter inhaltlichen und methodologischen Aspekten gerecht werden. Am Beispiel der dritten Konvergenzstudie werden die methodischen Zugänge bei der Erhebung (Kontaktinterview und Intensivinterview mit Narrationsanreizen, Erfassung von Kontextinformationen) sowie bei der Auswertung vorgestellt (Herausarbeitung typischer Muster der konvergenzbezogenen Medienaneignung durch Einzelfallstudien). Als Leitlinien für ihr forschungsmethodisches Vorgehen heben die Autorinnen «Kontextorientierung», «sinnliche und symbolische Dimensionen» sowie «Exemplarität» des Medienhandelns hervor. 
Mehrere Beiträge gehen auf die Frage ein, welche besonderen Bedingungen mit einer Erforschung des Internets verknüpft sind. Winfried Marotzki betont in seinem Beitrag den kulturellen Charakter des Internets und stellt im Anschluss daran die Charakteristik jener Trends dar, welche mit dem Begriff des Web 2.0 verbunden sind. Methodisch favorisiert er zur Erforschung dieses neuen Raumes diskursanalytische Verfahren in der Tradition wissenssoziologischen Denkens. Diskurse sieht er in diesem Zusammenhang als Artikulation von Erfahrungsräumen innerhalb eines multimodalen Ausdruckskontinuums.

Martina Schuegraf stellt in ihrem Beitrag «Das Surfgramm als grafische Darstellung von Surfwegen im Internet» ein methodisches Instrument vor, das die Autorin nicht als ein technisch komplexes und cobjektives Daten lieferndes Methodeninstrumentarium, sondern als eine ergänzende Methode versteht. Gegenstand ihres Forschungsprojekts ist der medienkonvergente Umgang von Jugendlichen mit in Deutschland empfangbaren Musikkanälen und den dazugehörigen Internetseiten. In der Verknüpfung von thematisch fokussierten Interviews (mit erzählgenerierenden Eingangsfragen) und gemeinsamen Surfen im Internet (in Verbindung mit der Methode des «lauten Denkens») werden Daten erhoben und anschliessend im Rahmen einer «doppelten Rekonstruktion» ausgewertet. Diese Rekonstruktion umfasst die grafische Visualisierung des Surfwegs der befragten Nutzer/innen. Der Beitrag illustriert das methodische Vorgehen anhand von zwei Beispielen.

An der Schnittstelle zwischen mediendidaktischen und schulbezogenen Beiträgen argumentieren die drei Beiträge von Kerstin Mayrberger, Renate Schulz-Zander und Birgit Eickelmann und Claudia Zentgraf u.a. Sie zeigen ein breites Spektrum von möglichen Zugängen zum Schulfeld bzw. zur Erforschung von Schulentwicklungsprozessen aus der Perspektive qualitativer Forschung auf.

Renate Schulz-Zander und Birgit Eickelmann gehen von den Ergebnissen der internationalen und nationalen qualitativen IEA-Studie SITES Modul 2 aus und beschreiben nach einem ausführlichen Projektaufriss die Folgeuntersuchung zu SITES M2 eine qualitative und quantitative Studie auf der Basis von Fallstudien. Ziel der Studie ist es, mit den Erhebungsinstrumenten sowohl den Entwicklungsprozess zu rekonstruieren wie die erforderlichen Informationen über den Grad der Nachhaltigkeit der Implementierung zu liefern. In der Anlage verbindet das Projekt quantitative und qualitative Methoden, indem leitfadengestützte Interviews, problemzentrierte Interviews und standardisierte Befragungen mit Fragebögen kombiniert werden.

Claudia Zentgraf u.a. fordern in ihrem Beitrag eine «Neuorierentierung der wissenschaftlichen Begleitforschung» und stellen den Ansatz einer lernenden Konzeption für die wissenschaftliche Begleitung und Evaluation zur Diskussion. Dieser bezieht sich auf das Projekt "Schule interaktiv», welches mit mehreren Schulen in zwei Bundesländern durchgeführt wurde. Die wissenschaftliche Begleitung ging dabei von der Relevanz der neuen Medien für Prozesse der Schulentwicklung aus. 
In diesem Zusammenhang stellen sie eine Evaluationskonzeption vor, die Impulse für die schulspezifischen Entwicklungsprozesse im Hinblick auf die übergeordneten Ziele des Projekts geben soll und dem Aufbau einer Evaluations- und Feedbackkultur dient. Im Einklang mit neueren Entwicklungen der Schulforschung verbinden sie dabei Fremd- und Selbstevaluation ebenso wie qualitative und quantitative Fragen (Leitfadeninterview und Fragebogen).

Gegenüber diesen stark auf die Umsetzung von Innovationen bezogenen Ansätzen geht es Kerstin Mayrberger primär um die methodologische Fundierung rekonstruktiver Ansätze. Insbesondere setzt sich die Autorin mit dem Ansatz der objektiven Hermeneutik nach Oevermann auseinander und versucht dessen Potenziale und Grenzen herauszuarbeiten. Dies geschieht im Rahmen eines Forschungsprojektes zu Unterrichtsprozessen in der Grundschule. Kerstin Mayrberger sieht in der Aufnahme des Oevernmannschen Forschungskonzeptes die Chance für mediendidaktische Fragestellungen, vom Material ausgehend «Neues» zu entdecken und dabei latente Sinnstrukturen zu erfassen bzw. objektive Handlungsstrukturen in Lernprozessen herauszuarbeiten. 